\title{
Study of the combinatorial impact of empathy and emotion on the processing of conflicts of interest with the event-related potential technique
}

This article was published in the following Dove Press journal:

Neuropsychiatric Disease and Treatment

29 June 2017

Number of times this article has been viewed

\section{Xiaoli He'}

Ni Zhang ${ }^{2}$

'Department of Psychology, Ningxia University, Yinchuan, ${ }^{2}$ Center of Mental Health Education for College Students, Weifang Medical University, Weifang, China
Correspondence: Xiaoli He Department of Psychology, Ningxia University, 489 West Helanshan Road, Xixia, Yinchuan, Ningxia, 750021, China Tel +86 I829520 0l7|

Email hexl@nxu.edu.cn

Ni Zhang

Center of Mental Health Education for College Students, Weifang Medical University, Baotong West Street, Weicheng Qu, Weifang, Shandong 261053, China

Tel +86 I876576 6530

Email zhangni223@I26.com
Objectives: Studies have found that empathy is important in moral development and violence suppression, and emotion also affects empathy. However, the combinatorial effect of emotion and empathy on the processing of conflicts is not known.

Materials and methods: A total of 44 undergraduate students ( 23 in low-empathy group and 21 in high-empathy group) were enrolled in this study. They were subjected to positive, negative, and neutral emotion evoking, as well as conflicting or nonconflicting proposals. Event-related potential technology was used to study the combinatorial effects of empathy and emotion on the processing of conflict of interest.

Results: We found that under the influence of a positive emotion, both low- and high-empathy groups exhibited lower rejection rates. In the context of conflict, individuals in the high-empathy group showed fewer refusals under positive emotion. In the low-empathy group, there was no significant difference between responses to different emotions, but conflicting proposals induced more negative medial frontal negativity than nonconflicting proposals. Individuals in the low-empathy group showed different late positive potentials when responding to different types of proposals under both neutral and negative emotions, whereas those in the high-empathy group only showed different late positive potentials responding to different types of proposals under negative emotion.

Conclusion: Our results indicate that under positive emotion, individuals with low empathy show less difference in processing either conflicting or nonconflicting proposals, whereas under negative emotion, individuals with high empathy show enhanced motivation toward nonconflicting proposals.

Keywords: empathy, conflicts of interest, emotion, event-related potential, late positive potentials, medial frontal negativity

\section{Introduction}

Conflict refers to friction, disagreement, or discord, and is one social interaction commonly seen during interpersonal communications in human society. With the development of cognitive neuroscience, many researchers have begun to focus on in-depth exploration of the neural mechanisms of conflict and the processing of it. A common approach for experimental study of the processing of conflicts of interest is the ultimatum game (UG). ${ }^{1}$

Empathy refers to the understanding of emotions and mental status of others and speculation on other people's behavior; it is an important positive development factor in human interactions. It is generally believed that empathy will increase prosocial behavior and reduce or prevent antisocial behavior, such as assault and 
juvenile delinquency. Under certain conditions, empathy can inhibit aggressive behavior. However, it is unknown whether empathy can inhibit the conflict with general intensity and what the neuropsychological mechanisms underlying the effect of empathy on conflict are.

Emotion includes the mental and physiological phenomena that change constantly, reflecting the body's adaptive model responding to a changing environment. ${ }^{2}$ In conflicts of interest, emotion also has an impact on the processing of conflict. The impacts of emotion on empathy are mainly manifested in the promotive effect of positive emotions and the inhibitory effect of negative emotions. ${ }^{3}$ Carlson et $\mathrm{al}^{4}$ also found through meta-analysis that positive emotions help to increase helping behavior, while the relationship between negative emotions and helping behavior was also influenced by attention directivity, responsibility, arousal, and other factors. Currently, there are few reports studying the combinatorial effect of emotion and empathy on conflict and exploring its neuropsychological mechanisms.

Event-related potential (ERP) is measured by electroencephalography (EEG) and is the measured brain response that is the direct result of a specific sensory, cognitive, or motor event. It has become one of the most widely used methods in cognitive neuroscience research to study the physiological correlates of sensory, perceptual, and cognitive activity associated with processing information..$^{5-8}$ Some researchers have even used ERP to study the development of empathy in early childhood. ${ }^{9}$ Some other researchers have used ERP to study the underlying mechanism of processing of conflicts, ie, to explore in depth the reason for the test-takers to reject an unfair proposal (with conflict of interest) in the UG test. ${ }^{10,11}$ These ERP studies have shown that the reason for responders to reject the proposal with conflict of interest mainly relies on mediofrontal negativity (MFN): proposals with conflicts of interest induced more negative MFN than proposals without conflicts of interest. ${ }^{10,11}$

In the present study, we used the UG model to create conflict-of-interest situations, and then to study the effect of empathy and emotion on the processing of conflicts of interest under such situations with ERP technology. Our hypothesis is that different emotions will affect empathy and subsequently affect the processing of conflict of interests; specifically in a positive emotion, there will be no significant difference in ERP components between the low-empathy and high-empathy groups, whereas in a negative emotion, there will be significant differences.

\section{Materials and methods Subjects}

The Chinese version of the Interpersonal Reactivity Index (IRI-C) was created by a Taiwanese scholar, based on the original English version of the Interpersonal Reactivity Index. The IRI-C is a self-rating scale and contains 22 questions divided into four categories: perspective consideration, empathy concern, fantasy, and personal distress. IRI-C questionnaires were distributed to 250 undergraduate students from non-psychology and non-economics majors, and 238 valid answers to the questionnaires were collected. The subjects' empathy scores were ranked from low to high, and the average empathy score was 52.87, with a standard deviation of 14.45 . Subjects with empathy scores higher than the average score with one standard deviation ( $\geq 68$ ) were classified as the highempathy group, whereas those with empathy scores lower than average score with one standard deviation $(\leq 38)$ were classified as the low-empathy group. ERP studies were then performed in each subject, as described in detail in the following section. Eight subjects were excluded from the statistical analysis, due to big artifacts in ERP, and six subjects were excluded from the statistical analysis as the effective trials number did not reach $70 \%$ of the total number after removal of artifacts. A flowchart of the experiment design is shown in Figure 1. All subjects were right-handed, had normal vision or corrected visual acuity, were physically healthy, had no neurological or psychiatric history, and had not participated in similar experiments. All subjects signed the informed consent, and this study was approved by the ethics committee of Ningxia University.

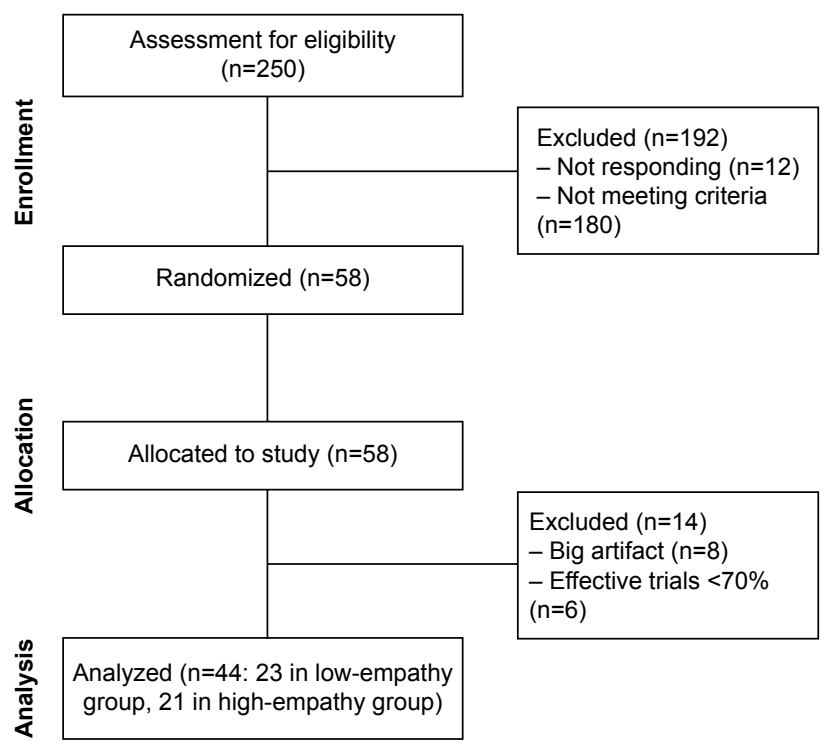

Figure I Flowchart of study design. 


\section{Experimental design}

A mixed-design, 3 (emotion: positive/negative/neutral) $\times 2$ (empathy: high empathy/low empathy) $\times 2$ (proposal type: conflicting/nonconflicting) was used in this study, with empathy as a between-subject variable and emotions and proposal type as within-subject variables. The experimental stimuli were 50 pictures of positive, negative, and neutral emotions, respectively, that were selected from the standardized Chinese Affective Picture System (CAPS). ${ }^{12}$ The selected pictures of positive and negative emotions were matched on arousal and potency to ensure that there was no difference in arousal between the positive and negative emotion pictures and the difference in potency was significant. Adobe Photoshop software was used to modify the pixelation, size, background, brightness, contrast, and color saturation of the images, in order to ensure the consistency of the stimuli. The emotion self-rating scale associated with CAPS pictures was used to evaluate the subjects' emotional feelings responding to the CAPS pictures in five aspects (disgust, anger, calm, euphoria, joy) with grade ratings of 1-7: 1 showed the weakest emotional intensity, and 7 represented the strongest emotional intensity. The dependent variables were reaction time, rejection rates, and EEG ingredients at the decision stage.

\section{Experimental procedure}

After the high- and low-empathy groups were sorted out, all subjects were subjected to positive, negative, and neutral emotion evoking. Each subject completed the experiments independently. To eliminate gender confounding, male lab assistants partnered with male subjects, and female lab assistants partnered with female subjects. Before the formal test, subjects were told to sit in a comfortable position; to hold their head, face, and body posture as stable as possible; and to focus on the task with the index fingers of both hands on computer keys lightly.

An improved version of the UG task was used in the formal experiment. ${ }^{11}$ There were three blocks in the whole experimental task, with only one emotion picture in each block, for a total of 100 trials. A positive image was presented twice to induce positive emotions, a negative image was presented twice to induce negative emotions, and a neutral image was presented twice to induce a neutral emotion. Each of the 50 kinds of proposals was presented twice in each block. All participants were asked to complete three tasks, with each task randomly presented; the resting time between tasks was freely controlled by the study subject, and during

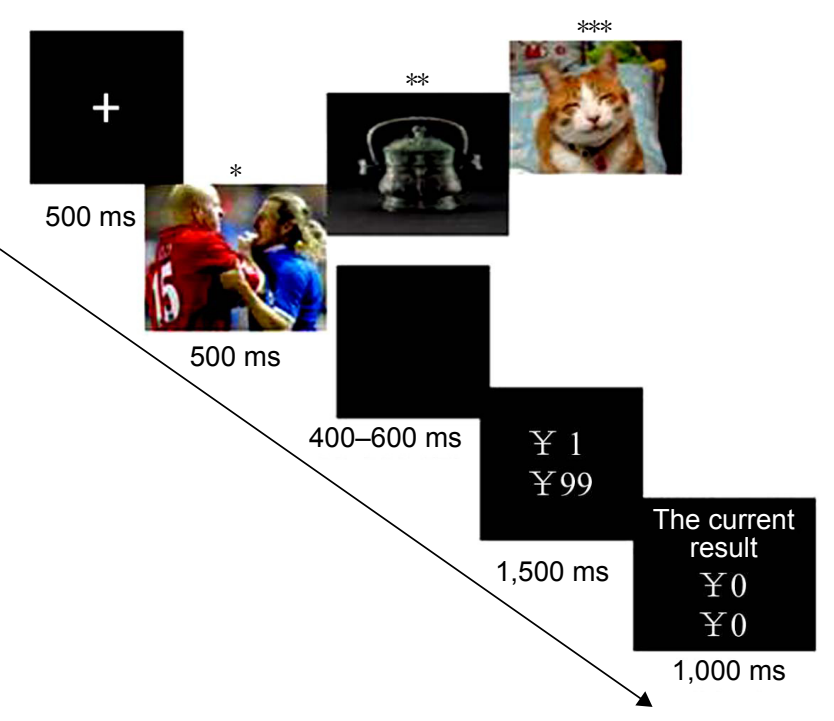

Figure 2 Flowchart of a single round of the experiment. Note: Examples of $*_{\text {negative, }}^{* *}$ neutral, and ${ }^{* * *}$ positive images.

this time, the emotion self-rating scale was performed. A flowchart for a single round of the experiment is shown in Figure 2. Each experiment lasted about 25 minutes. The recess time between each experiment was controlled by the subjects themselves, but they were asked to take enough rest and remain calm before the start of each experiment so that factors that may have influenced brain activity, such as tiredness and sleepiness, were excluded.

\section{ERP recording}

An EEG acquisition and analysis system with SynAmps2 amplifiers from Compumedics NeuroScan (Melbourne, VA, Australia) was used to record EEG signals with Quik-Cap electrode caps, which have a 64-lead $\mathrm{Ag} / \mathrm{AgCl}$ electrode expanded in accordance with the international 10-20 system. The left mastoid (M1) was used as the reference electrode during the experiment, whereas for offline analysis, the average of the bilateral mastoid was used as reference. With the forehead grounded, left and right lateral orbital horizontal electrooculogram and left-eye vertical electrooculogram were recorded simultaneously.

Scan 4.5 software was used to perform offline data analysis. The EEG data from -100 to $1,000 \mathrm{~ms}$ were epoch, and the baseline was the $100 \mathrm{~ms}$ before stimulus presentation. Artifacts caused by eye blinking, eye movement, or body shaking were eliminated. The filter band pass was $0.05-30 \mathrm{~Hz}$, and artificial signals with amplitudes greater than $\pm 100 \mu \mathrm{V}$ were excluded. According to observation of the data, periods between 250 and $400 \mathrm{~ms}$ and between 450 and $600 \mathrm{~ms}$ were used as the analysis window. The 12 electrodes - F3, Fz, 
F4, FC3, FCz, FC4, C3, Cz, C4, CP3, CPz, and CP4 - were used as analysis electrodes, and the amplitude of each time window was the dependent variable. After EEG recording, questionnaires of self-rating of the intensity of the conflict were distributed to the study subjects.

\section{Statistical analysis}

One-way repeated-measures analyses of variance (ANOVAs) and paired $t$-tests were performed using SPSS software, ie, 2 (empathy: high/low) $\times 3$ (emotion: positive/neutral/ negative) $\times 2$ (proposal type: conflicting/nonconflicting) $\times 12$ (electrodes). The results of the ANOVA were corrected with the Greenhouse-Geisser method. $P<0.05$ was considered statistically significant.

\section{Results}

There were 23 subjects in the low-empathy group (mean age 20.00 years, SD 0.96) and 21 in the high-empathy group (mean age 19.73 years, SD 0.88). There was no significant difference in demographics between the two groups.

\section{Verification of emotion-evoking effect of Chinese affective picture system pictures}

As shown in Table 1, there was no difference in arousal between the pictures of positive and negative emotion, whereas the difference in potency was significant. Based on the methods described in the existing literature, ${ }^{13,14}$ the emotions and emotional self-rating of every subject toward the Chinese affective picture system pictures in three blocks were assessed. Through one-way repeated-measures ANOVA, in terms of scores in emotional feelings, there was a significant main effect of emotion $\left(F_{2,40}=12.05, P<0.001\right)$, and paired $t$-test results showed that differences between the two groups were significant $\left(t_{20}=2.98, P<0.01 ; t_{20}=4.7, P<0.001 ; t_{20}=2.03\right.$, $P=0.056)$. In terms of scores in emotions, there was also a significant main effect of emotion $\left(F_{2,40}=12.68, P<0.001\right)$, and paired $t$-test results also showed significant differences between the two groups $\left(t_{20}=2.94, P<0.01 ; t_{20}=4.77, P<0.001\right.$; $\left.t_{20}=2.29, P<0.05\right)$. These data indicate that it is valid to use emotional pictures to induce different emotions.

Table I Values of potency and arousal of pictures of positive and negative emotions

\begin{tabular}{llll}
\hline Dimensions & $\begin{array}{l}\text { Pictures of } \\
\text { positive emotions }\end{array}$ & $\begin{array}{l}\text { Pictures of } \\
\text { negative emotions }\end{array}$ & t-value \\
\hline Potency & $6.43 \pm 0.41$ & $5.15 \pm 0.24$ & $19.26 * * *$ \\
Arousal & $5.40 \pm 0.31$ & $5.17 \pm 0.25$ & 4.17 \\
\hline Notes: $* * * P<0.001$ & Valos are mean \pm standard deviation
\end{tabular}

Notes: $* * * p<0.001$. Values are mean \pm standard deviation.

\section{Behavioral outcomes}

A simple effect test showed that there were significant differences in reaction time to different types of proposals under positive emotion $\left(F_{1,20}=5.4, P<0.05\right)$ and neutral emotion $\left(F_{1,20}=8.53, P<0.01\right)$, whereas under negative emotion there was no significant difference in reaction time to different types of proposals $\left(F_{1,20}=0.75, P>0.05\right)$. Compared with those under neutral emotion, the reaction time to conflicting proposals under positive emotion was shorter than to nonconflicting proposals, and compared with those under neutral emotion, the reaction time to conflicting proposals under negative emotion was similar to nonconflicting proposals. There was no significant difference in terms of the main effect of other variables or interactions among the variables.

Under positive emotion, the rejection rate (mean $43.45 \%$, SD $15.62 \%$ ) was significantly lower than the other two conditions. The rejection rate of conflicting proposals (mean $84.80 \%$, SD 16.67) was significantly greater than nonconflicting proposals (mean 19.33\%, SD 14.40), and there was significant interaction between emotion and proposal type $\left(F_{2,38}=5.04\right.$, $P<0.05)$. Simple effects test results showed that the rejection rate of conflicting proposals was significantly affected by emotions $\left(F_{2,40}=14.85, P<0.001\right)$ : with positive emotion, the rejection rate was the lowest (mean $71.55 \%$, SD 24.97), whereas the rejection rate of nonconflicting proposals was not significantly affected by emotions $\left(F_{2,40}=3.01, P>0.05\right)$. There were significant interactions among group, emotion, and proposal type $\left(F_{2,38}=5.69, P<0.01\right)$. Simple effects test results showed that in the high-empathy group, the rejection rate of conflicting proposals was significantly influenced by emotions $\left(F_{2,38}=22.03, P<0.001\right)$ : with positive emotion, the rejection rate was the lowest (mean 65.68\%, SD 22.39), whereas the rejection rate of nonconflicting proposals was not affected by emotions $\left(F_{2,38}=1.41, P>0.05\right)$. In the low-empathy group, the rejection rate of conflicting or non-conflicting proposals was not significantly affected by emotions $(P>0.05)$.

\section{EEG results}

As shown in Figure 3, there was a significant negative deflection of wave amplitude between 250 and $400 \mathrm{~ms}$ in both the high- and low-empathy groups. Further analysis showed that in the wave amplitude of MFN, the main effect of emotion was significant $\left(F_{2,38}=7.15, P<0.05\right)$, and the wave amplitude of MFN induced by positive emotion was the most negative (mean $-1.30 \mu \mathrm{V}, \mathrm{SD} 3.11$ ). The main effect of proposal type was also significant $\left(F_{1,19}=8.04, P<0.01\right)$, and the wave amplitude of MFN induced by conflicting proposals (mean $-0.74 \mu \mathrm{V}, \mathrm{SD} 3.25$ ) was more negative than that 

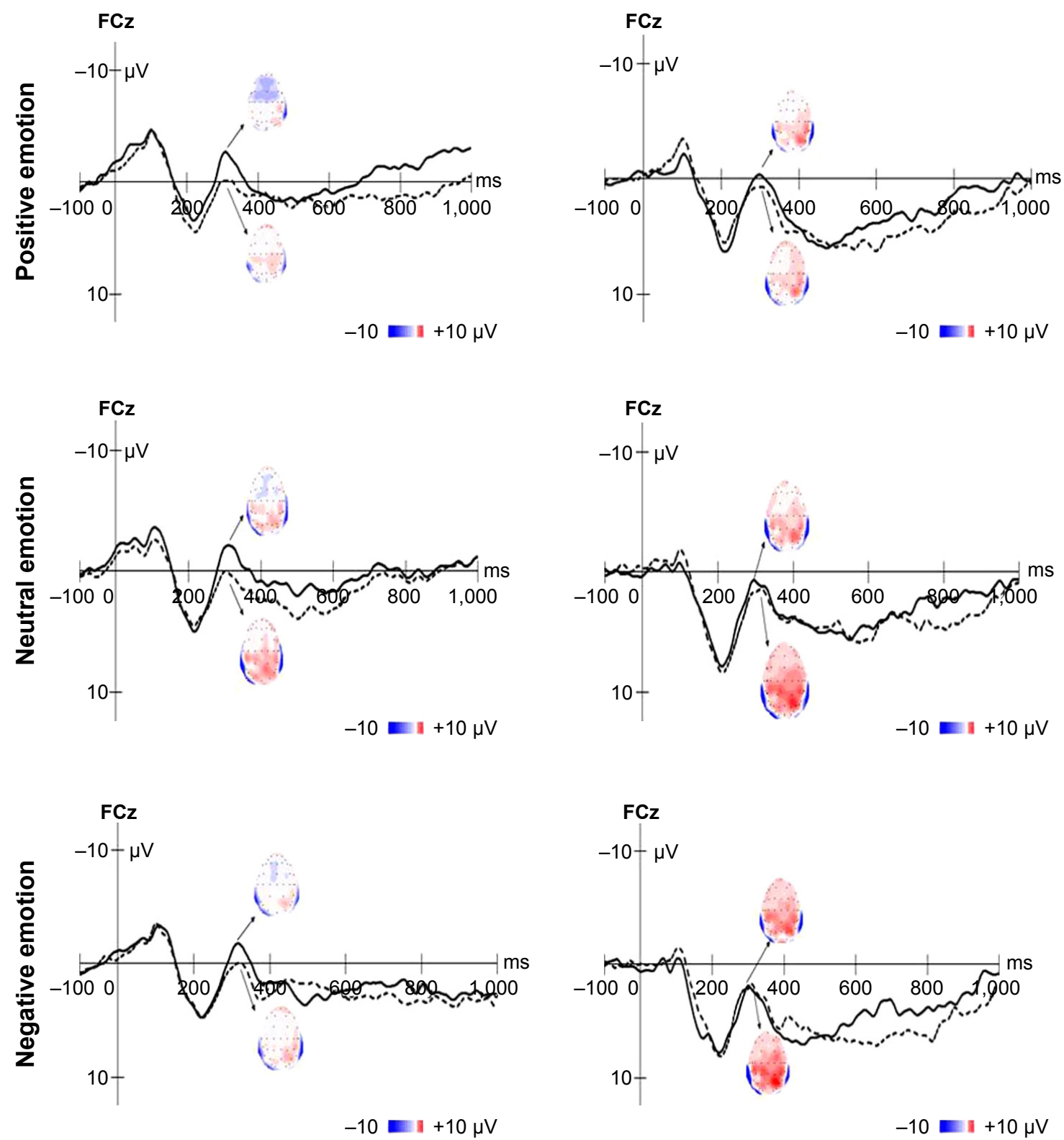

Low-empathy group

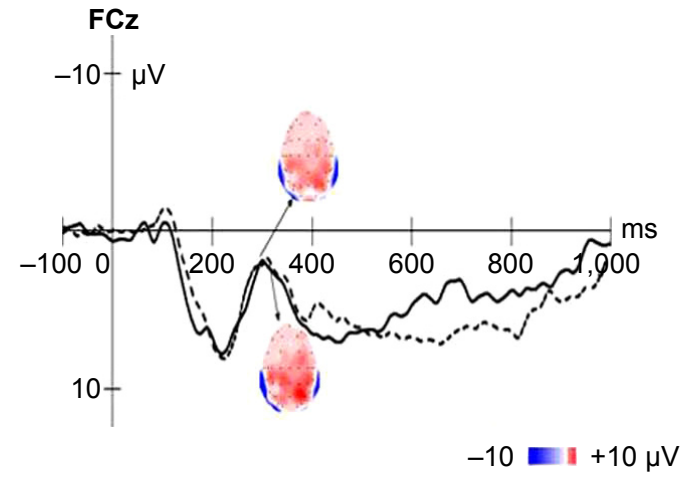

High-empathy group

Conflicting proposal --- Nonconflicting proposal

Figure 3 Average ERP waveforms and brain mapping at FCz points of high- and low-empathy groups under different emotional conditions. Abbreviations: ERP, event-related potential; FCz, frontal center zone.

induced by nonconflicting proposals (mean $0.07 \mu \mathrm{V}, \mathrm{SD}$ 3.32). The main effect of electrode points was significant $\left(F_{11,209}=5.51, P<0.05\right)$.

There were significant interactions among empathy, emotion, and electrode point $\left(F_{22,418}=2.84, P<0.05\right)$, ie, in the low-empathy group, there was no significant difference between the wave amplitudes of MFN in the frontal and central regions under different emotions $(P>0.05)$, whereas in the high-empathy group, there was a significant difference between the wave amplitudes of MFN in the frontal and central regions (F3, FC3, FCz, C3, Cz, C4, CP3, CPz, CP4) under different emotions $(P<0.05)$. Repeated-measures ANOVA (3 [emotion] $\times 2$ [proposal type] $\times 12$ [electrode points]) analysis of the mean wave amplitude of different empathy groups between 250 and $400 \mathrm{~ms}$ was further performed.

Results showed that in the low-empathy group, the main effect of emotion was not significant $\left(F_{2,18}=1.58, P>0.05\right)$, the main effect of proposal type was significant $\left(F_{1,9}=7.15\right.$, 
$P<0.05)$, MFN induced by conflicting proposals was more negative that that induced by nonconflicting proposals, and there were no significant interactions among emotion, proposal type, and electrode point $\left(F_{22,198}=1.89, P>0.05\right)$; on the contrary, in the high-empathy group, the main effect of emotion was significant $\left(F_{2,20}=8.93, P<0.001\right)$, the wave amplitude of MFN induced by positive emotion was the most negative (mean $-0.65 \mu \mathrm{V}$, SD 2.42), the main effect of proposal type was not significant $\left(F_{1,10}=1.89, P>0.05\right)$, and there was no significant interaction among emotion, proposal type, and electrode point $\left(F_{22,220}=0.97, P>0.05\right)$.

Repeated-measures ANOVA of mean wave amplitudes between 450 and $600 \mathrm{~ms}$ was performed, and the main effect of emotion was significant $\left(F_{2,38}=5.31, P<0.05\right)$. The late positive potential (LPP) induced by different proposal types under negative emotion was the biggest. The main effect of electrode point was significant $\left(F_{11,209}=10.65, P<0.001\right)$, wave amplitudes of LPP increased sequentially from the frontal region to the central region, the amplitude of LPP of the central electrode was the biggest, and there was a significant interaction between electrode point and empathy group $\left(F_{11,209}=3.21, P<0.05\right)$. There was no significant difference in mean wave amplitudes of LPP on electrode points in the frontal or central region in the low-empathy group $\left(F_{11,209}=1.60\right.$, $P>0.05)$, whereas there was a significant difference in the high-empathy group $\left(F_{11,209}=12.78, P<0.001\right)$. There was a significant interaction between emotion and proposal type $\left(F_{2,38}=3.94, P<0.05\right)$. There was a significant difference in LPP wave amplitudes induced by different proposal types under neutral emotion $\left(F_{1,20}=7.73, P<0.05\right)$, whereas there was no significant difference under other emotional conditions $(P>0.05)$. There were significant interactions among group, emotion, and proposal types $\left(F_{2,38}=10.31, P<0.001\right)$.

Simple effects tests showed that in the low-empathy group, there were significant differences in LPPs induced by different proposal types under neutral $\left(F_{1,19}=11.03\right.$, $P<0.01)$ and negative $\left(F_{1,19}=14.57, P<0.01\right)$ emotions, and nonconflicting proposals induced larger LPP than conflicting proposals under neutral emotion, whereas under negative emotion, conflicting proposals induced larger LPP than nonconflicting proposals, and there was no significant difference under positive emotion $\left(F_{1,19}=1.22, P>0.05\right)$.

In the high-empathy group, there was a significant difference only between LPPs induced by different proposal types under negative emotions $\left(F_{1,19}=9.85, P<0.01\right)$, and nonconflicting proposals induced larger LPPs than conflicting proposals, whereas there was no significant difference under the other two emotional conditions $(P>0.05)$. Within this time window, the main effect of group difference was not significant
$\left(F_{1,19}=3.61, P>0.05\right)$, and there were no significant interactions among other variables either $(P>0.05)$ (Figure 4$)$.

\section{Intensity of conflict}

Statistical analysis of self-rating scores of intensity of conflict showed no significant difference between the two groups, which indicates that the test material was effective in mimicking the situation of conflict of interest.

\section{Discussion}

In terms of the impact of emotion on empathy, previous research showed that positive emotions could engender more positive social cognition in individuals such that the kindness and cooperation nature of humankind were more appreciated, and thus, helping behavior was promoted. Under a pleasant emotion, an individual is prone to making a positive judgment and response toward external stimuli; on the contrary, under a negative emotion, the individual is more inclined to make negative judgments and choices toward external stimuli. ${ }^{15}$

In this study, after the addition of emotional factors, we also found that under both positive and neutral emotions, the reaction times to conflicting proposals of both high- and lowempathy groups were shorter than those toward nonconflicting proposals. The rejection rates of the two groups with positive emotion were significantly lower than under the other two conditions, and the rejection rate of the two groups of conflicting proposals was significantly higher than nonconflicting proposals. Furthermore, in the high-empathy group, the rejection rate of conflicting proposals under positive emotions was the lowest, whereas the rejection rate of nonconflicting proposals was not significantly affected by emotions. In the low-empathy group, the rejection rate of different types of proposals was not significantly affected by emotions. The results of this study further showed that compared with the low-empathy group, individuals in the high-empathy group were more sensitive in emotional feeling, such that under the impact of a positive emotion, the high-empathy group showed different responses under different conflict situations, whereas there was no difference in the low-empathy group, likely due to the low sensitivity in emotional feeling.

The ERP data from our study showed that there were significant differences in brain electrical activities within the $250-400 \mathrm{~ms}$ range responding to different types of proposals under different emotions between the low- and high-empathy individuals. Among them, the high-empathy group was significantly influenced by emotional factors, especially under positive emotions, and the wave amplitude of MFN was the most negative. It has been reported that positive emotions can enhance the sensitivity of individual cognition, ${ }^{16}$ as well 

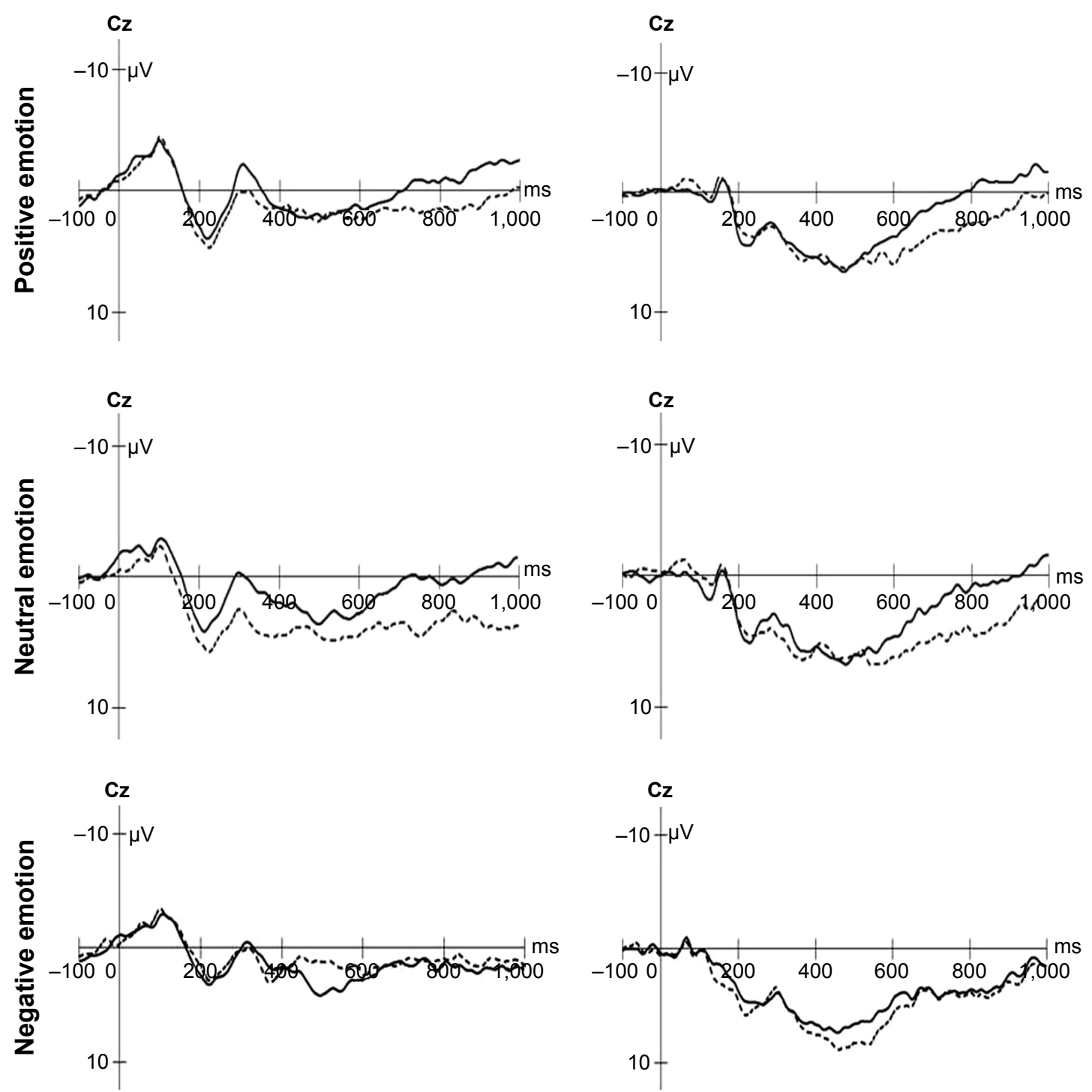

Low-empathy group

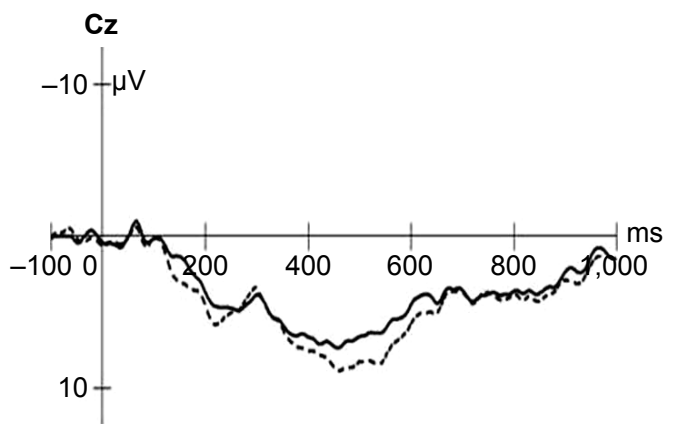

High-empathy group

Conflicting proposal -- Nonconflicting proposal

Figure 4 Average ERP waveforms at $\mathrm{Cz}$ points of high- and low-empathy groups under different emotional conditions. Abbreviations: $\mathrm{Cz}$, center zone; ERP, event-related potential.

as promote other cognitive functions, such as attention and problem solving. ${ }^{17}$ Although the low-empathy group was not significantly affected by different emotions, ie, conflicting proposals still induced more negative MFN than nonconflicting proposals, this result may indicate that individuals in the low-empathy group were more purposeful in task selection, ie, maximizing own benefits, and thus, they allocated more cognitive resources to process the proposed information itself, and when conflicting proposals appeared, the result can be effectively extracted and characterized to show the difference in the MFN. In addition, research has shown that MFN not only reflects individual cognitive appraisal of a situation, but under a positive emotion it is also beneficial to self-awareness of different scenarios. ${ }^{18}$ We did not find that individuals with high empathy were more easily able to distinguish between different types of conflict situations under positive emotions. The reason may be that though individuals with high empathy had enhanced awareness of different tasks, more perspective-taking processes were also performed, and when more cognitive resources were occupied by perspective-taking process, fewer cognitive resources would be allocated to conflicting proposal processing, thus leading to nonsignificant differences in MFN of the highempathy group when responding to different types of proposals. To some extent, this indicates that empathy and emotion play an inhibitory role in conflict processing. Furthermore, 
in the high-empathy group, the difference in MFN amplitude responding to different emotions was mainly located in the frontal and central regions of the brain, whereas in the low-empathy group there was no difference between MFN amplitudes in the frontal and central regions under different emotions. Generally, it is believed that wave amplitude reflects the strength of brain activity. ${ }^{2}$ Our study results suggest that to a certain degree, compared with the low-empathy group, brain activity in the high-empathy group was higher in response to different types of conflict situations.

From the ERP data within 450-600 ms, we found that there were significant differences in ERP between the different empathy groups when responding to different types of proposal under different emotions. In the low-empathy group, there were significant differences in LPPs induced by different types of proposal under neutral and negative emotions, but no differences under positive emotion, which indicates that people with low empathy will pay more attention to other people's situations and psychological states under positive emotions, ${ }^{19}$ and likely enhance perspective-taking processing, and thus weaken the processing of information of different types of proposals. Under a neutral emotion, they still have a higher arousal to nonconflicting proposals and a stronger motivation, whereas under a negative emotion, higher individual physiological arousal and negative feelings are induced, ${ }^{20}$ such that the individual will pay more attention to conflicting proposals. In the high-empathy group, there were differences in LPPs induced by different types of proposals only under negative emotions, which may indicate that people with high empathy pay less attention to other people's situations and psychological states under negative emotions. ${ }^{19}$ They pay more attention to the pros and cons of their own interests, the tendency to seek advantages and avoid disadvantages is exposed, and the processing of motivation to nonconflicting proposals is enhanced, and thus, greater LPP was induced.

The weakness of our study is that the sample size is relatively small, and heterogeneity of our sample pool is not that great. We are planning to recruit more people of different socioeconomic statuses and age groups in future to perform a more thorough study.

\section{Conclusion}

Our results indicate that under positive emotion, individuals with low empathy show less difference in processing conflicting and nonconflicting proposals, whereas under negative emotion, individuals with high empathy show enhanced motivation toward nonconflicting proposals, confirming the combinatorial role of empathy and emotion in the processing of conflict of interest.

\section{Acknowledgment}

The study was approved by Ningxia University Scientific Research Project (number NGY2016046) and Ningxia Philosophy and Social Science Major Innovation Project (number SKZD2017005).

\section{Disclosure}

The authors report no conflicts of interest in this work.

\section{References}

1. Güth W, Schmittberger R, Schwarze B. An experimental analysis of ultimatum bargaining. J Econ Behav Organ. 1982;3(4):367-388.

2. Luo YJ, Huang YX, Li XY, Li XB. Effects of emotion on cognitive processing: series of event-related potentials study. Adv Psychol Sci. 2006;14(4):505-510.

3. Eisenberg N. Emotion, regulation, and moral development. Annu Rev Psychol. 2003;51:665-697.

4. Carlson M, Charlin V, Miller N. Positive emotion and helping behavior: a test of six hypotheses. J Pers Soc Psychol. 1988;55(2):211-229.

5. Suzuki Y, Galli L, Ikeda A, Itakura S, Kitazaki M. Measuring empathy for human and robot hand pain using electroencephalography. Sci Rep. 2015;5:15924.

6. Troup LJ, Bastidas S, Nguyen MT, Andrzejewski JA, Bowers M, Nomi JS. An event-related potential study on the effects of cannabis on emotion processing. PloS One. 2016;11(2):e0149764.

7. Cui F, Ma N, Luo YJ. Moral judgment modulates neural responses to the perception of other's pain: an ERP study. Sci Rep. 2016;6:20851.

8. Balconi M, Canavesio Y. Is empathy necessary to comprehend the emotional faces? The empathic effect on attentional mechanisms (eye movements), cortical correlates (N200 event-related potentials) and facial behaviour (electromyography) in face processing. Cogn Emot. 2016;30(2):210-224

9. Cheng Y, Chen C, Decety J. An EEG/ERP investigation of the development of empathy in early and middle childhood. Dev Cogn Neurosci. 2014;10:160-169.

10. Boksem MA, De Cremer D. Fairness concerns predict medial frontal negativity amplitude in ultimatum bargaining. Soc Neurosci. 2010; 5(1):118-128

11. Polezzi D, Daum I, Rubaltelli E, et al. Mentalizing in economic decisionmaking. Behav Brain Res. 2008;190(2):218-223.

12. Bai L, Ma H, Huang YX, Luo YJ. The development of native Chinese affective picture system: a pretest in 46 college students. Chin Ment Health J. 2005;19(11):719-722.

13. Harlé KM, Sanfey AG. Incidental sadness biases social economic decisions in the ultimatum game. Emotion. 2007;7(4):876-981.

14. Andrade EB, Ariely D. The enduring impact of transient emotions on decision making. Organ Behav Hum Decis Process. 2009;109:1-8.

15. Forgas JP. Affective influences on partner choice: role of emotion in social decisions. J Pers Soc Psychol. 1991;61(5):708-720.

16. Dreisbach G, Goschke T. How positive affect modulates cognitive control: reduced perseveration at the cost of increased distractibility. J Exp Psychol Learn Mem Cogn. 2004;30(2):343-353.

17. Chuang SC, Lin HM. The effect of induced positive and negative emotion and openness-to-feeling in student's consumer decision making. J Bus Psychol. 2007;22(1):65-78.

18. Wang YW, Huang L, Xu S, Yuan B, Xu YJ, Li HY. ERP time course of understanding private versus communicative intentions. Acta Psychol Sin. 2012;44(12):1618-1627.

19. Baron-Cohen $S$, Wheelwright $S$. The empathy quotient: an investigation of adults with Asperger syndrome or high functioning autism, and normal sex differences. J Autism Dev Disord. 2004;34(2):163-175.

20. Eisenberg N, Fabes RA, Schaller M, Carlo G, Miller PA. The relations of parental characteristics and practices to children's vicarious emotional responding. Child Dev. 1991;62(6):1393-1408. 
Neuropsychiatric Disease and Treatment

Dovepress

\section{Publish your work in this journal}

Neuropsychiatric Disease and Treatment is an international, peerreviewed journal of clinical therapeutics and pharmacology focusing on concise rapid reporting of clinical or pre-clinical studies on a range of neuropsychiatric and neurological disorders. This journal is indexed on PubMed Central, the 'PsycINFO' database and CAS,

and is the official journal of The International Neuropsychiatric Association (INA). The manuscript management system is completely online and includes a very quick and fair peer-review system, which is all easy to use. Visit http://www.dovepress.com/testimonials.php to read real quotes from published authors.

Submit your manuscript here: http://www.dovepress.com/neuropsychiatric-disease-and-treatment-journal 